Published in final edited form as:

Clin Nutr ESPEN. 2017 February ; 17: 18-21. doi:10.1016/j.clnesp.2016.12.003.

\title{
Coffee consumption and calcified atherosclerotic plaques in the coronary arteries: The NHLBI Family Heart Study
}

\author{
Yash R. Patel ${ }^{a,}{ }^{*}$, Taraka V. Gadiraju ${ }^{a, b}$, R. Curtis Ellison ${ }^{c}$, Steven C. Hunt ${ }^{\mathrm{d}, \mathrm{e}}$, John Jeffrey \\ Carr $^{f}$, Gerardo Heiss $^{g}$, Donna K. Arnett ${ }^{\mathrm{h}}$, James S. Pankow', J. Michael Gaziano ${ }^{\mathrm{a}, \mathrm{j}}$, and Luc \\ Djousséa,j \\ aDivision of Aging, Department of Medicine, Brigham and Women's Hospital and Harvard Medical \\ School, Boston, MA, United States \\ bTulane Heart \& Vascular Institute, Tulane University School of Medicine, New Orleans, LA, \\ United States \\ 'Section of Preventive Medicine \& Epidemiology, Boston University, Boston, MA, United States \\ dDepartment of Genetic Medicine, Weill Cornell Medicine, Doha, Qatar \\ eCardiovascular Genetics Division, University of Utah School of Medicine, Salt Lake City, UT, \\ United States \\ fDepartment of Radiology, Cardiovascular Medicine and Biomedical Informatics, Vanderbilt \\ University Medical Center Nashville, TN, United States \\ gDepartment of Epidemiology, School of Public Health, The University of North Carolina at Chapel \\ Hill, NC, United States \\ hDivision of Epidemiology, University of Alabama, Birmingham, AL, United States \\ iDivision of Epidemiology, University of Minnesota, Minneapolis, MN, United States \\ jMassachusetts Veterans Epidemiology and Research Information Center (MAVERIC) and \\ Geriatric Research, Education, and Clinical Research Center (GRECC), Boston Veterans Affairs \\ Healthcare System, Boston, MA, United States
}

\section{SUMMARY}

Background \& aims-While a recent meta-analysis of prospective studies reported that coffee consumption is associated with a lower risk of cardiovascular disease mortality, limited and inconsistent data are available on the relation of coffee intake with subclinical disease. Thus, the

\footnotetext{
*Corresponding author. Division of Aging, Brigham and Women's Hospital, 1620 Tremont St. 3rd Floor, Boston, MA 02120, United States. Fax: +1 617525 7739. dryashpate121@ gmail.com (Y.R. Patel).

Disclosures/conflict of interests

None by any authors.

Author contributions

YRP and LD designed research; all authors conducted research; YRP and LD analyzed data and performed statistical analyses; YRP drafted and made substantial revisions to the manuscript and had primary responsibility for the final content; YRP, TVG, JMG, and LD reviewed and edited the manuscript for the content. JJC performed CAC measurements; RCE, DKA, JSP, GH, SCH obtained funding, collected data, and critically reviewed the paper for content. All authors provided critical revisions for content and had responsibility for the final content.
} 
aim of the present study was to see the association of coffee consumption with the prevalence of atherosclerotic plaque in the coronary arteries in NHLBI Family Heart Study.

Methods-In a cross-sectional design, we studied 1929 participants of the NHLBI Family Heart Study without known coronary heart disease. Coffee consumption was assessed by a semiquantitative food frequency questionnaire and coronary-artery calcium (CAC) was measured by cardiac computed tomography. We defined prevalent CAC as an Agatston score of $\geq 100$ and used generalized estimating equations to calculate prevalence ratios of CAC as well as a sensitivity analysis at a range of cutpoints for CAC.

Results-Mean age was 56.7 years and 59\% of the study subjects were female. In adjusted analysis for age, sex, BMI, smoking, alcohol, physical activity, field center, and energy intake, prevalence ratio (95\% CI) for CAC was 1.0 (reference), 0.92 (0.57-1.49), 1.34 (0.86-2.08), 1.30 (0.84-2.02), and 0.99 (0.60-1.64) for coffee consumption of almost never, <1/day, 1/day, 2-3/day, and $\geq 4$ cups/day, respectively. In a sensitivity analysis, there was no evidence of association between coffee consumption and prevalent CAC when CAC cut points of 0,50,150, 200, and 300 were used.

Conclusions-These data do not provide evidence for an association between coffee consumption and prevalent $\mathrm{CAC}$ in adult men and women.

\section{Keywords}

Coffee consumption; Epidemiology; Atherosclerosis; Nutrition; Coronary artery calcium

\section{Introduction}

Coffee is consumed by $63 \%$ of adults in United States with an average consumption of 2.9 cups/day [1]. In a recent meta-analysis of 21 prospective studies, coffee consumption was associated with a lower risk of cardiovascular disease mortality, with the lowest risk observed among consumers of 4 cups/day [2]. It is unclear whether coffee consumption influences subclinical disease.

Coronary artery calcium (CAC) detected by cardiac computed tomography (cardiac CT) is a subclinical marker of atherosclerosis, which predicts cardiovascular heart disease $[3,4]$. Limited and inconsistent data exist on the relation of coffee consumption with subclinical atherosclerosis. The Rotterdam coronary calcification study with 1570 adults found that coffee consumption of $>3$ to 4 cups per day was associated with lower risk of coronary calcification in women compared with three or less cups of coffee consumption per day [5]. In addition, in a cross sectional study of 25138 young and middle-age adults, moderate coffee consumption ( 3 to $<5$ cups per day) was associated with lower prevalence of subclinical coronary atherosclerosis [6]. However, in the CARDIA study with 5115 adults aged 18-30 years, no association was found between coffee consumption of >3-4 cups and coronary calcification [7].

We sought to test the hypothesis that moderate coffee consumption is associated with lower prevalence of atherosclerotic plaque in coronary arteries in National Heart, Lung, and Blood Institute Family Heart Study (NHLBI FHS). 


\section{Materials and methods}

\subsection{Study population}

The NHLBI FHS study was designed to identify and evaluate genetic and non-genetic determinants of coronary heart disease (CHD), preclinical atherosclerosis, and cardiovascular risk factors. The details of NHLBI FHS have been described in detail in prior publications [8,9]. A total of 5710 subjects underwent baseline clinical examination from 1993 to 1995 . Between 2002 and 2003, about one-third of these subjects were invited to participate in a clinical examination that included measurement of CAC with cardiac CT. In addition to this, Hypertension Genetic Epidemiology Network Study, an African-American center at University of Alabama, recruited subjects that underwent cardiac CT. Amongst 3370 subjects who had data on cardiac CT, 980 subjects did not have data on coffee consumption at baseline evaluation. 279 subjects were excluded for prevalent CHD, 19 subjects were non-white. The final sample size for current analyses was 1929. Each subject gave informed consent and the study protocol was reviewed and approved by each of the participating institutions.

\subsection{Assessment of coffee consumption}

Dietary information was collected through a staff-administered semi-quantitative food frequency questionnaire developed by Willett et al. [10]. The reproducibility and validity of the food frequency questionnaire have been documented elsewhere [11,12]. Each subject was asked the following question: "In the past year, how often on average did you consume caffeinated coffee?" (Item \#61 in the questionnaire forms). Possible responses were: almost never, 1-3/month, 1/week, 2-4/week, 5-6/week, 1/day, 2-3/day, 4-6/day, and >6/day. Due to sparse data, we combined adjacent categories while creating final exposure categories of almost never, <1/day, 1/day, 2-3/day, and $\geq 4$ cups/day for stable estimates.

\subsection{Measurement of calcified atherosclerotic plaque in the coronary arteries}

Cardiac CT examinations were obtained using General Electric Health Systems LightSpeed Plus and LightSpeed Ultra, Siemens Volume Zoom, or Philips MX 8000 machines. These examinations were performed using the same protocol as employed in the NHLBI's MultiEthnic Study of Atherosclerosis [13]. The details of cardiac CT examination have been reported in previous publications [9]. Cardiac CT images from all sites were sent electronically to the central CT reading center located at Wake Forest University Health Sciences, Winston Salem, NC, where trained CT analysts identified CAC in the epicardial coronary arteries using Agatston score. Agatston score refers to the amount of calcium detected by the scan based on the area and the density of the calcified plaques [14]. In this study, the sum of the vessel plaque is reported as the total CAC score. Total CAC scores from the first and second measured values were then averaged.

\subsection{Other variables}

Information on cigarette smoking and alcohol intake was obtained by interview during the clinic visit. Dietary information was obtained using a food frequency questionnaire. Level of physical activity during the previous year was estimated through self-reported data. Data on 
weight and height were collected with participants wearing scrub suits. Prevalent CHD was defined as a self-reported history of myocardial infarction, percutaneous transluminal coronary angioplasty, or coronary artery bypass graft. All variables used in these analyses were ascertained during the initial examination (1993-1995) except for CAC scores, which were obtained during a follow-up examination (2002-2003).

\subsection{Statistical analysis}

CAC was dichotomized into Agatston CAC score of 100+ versus less than 100, as described previously [9]. To correct for familial clustering, we used generalized estimating equations to calculate the prevalence ratios of CAC with corresponding 95\% confidence interval across categories of coffee consumption. Multivariate model was adjusted for age, sex, field center, body mass index (continuous), smoking (current smoker $\mathrm{Y} / \mathrm{N}$ ), alcohol intake (current alcohol intake $\mathrm{Y} / \mathrm{N}$ ), physical activity (quartiles of total MET-min/wk), and caloric intake (continuous). All analyses were completed using SAS, version 9.3 (SAS institute Inc, Cary, $\mathrm{NC}$ ). All $\mathrm{p}$-values were 2-tailed and significance level was set at an alpha of 0.05 .

\section{Results}

Of the total 1929 subjects, 59\% were female and the mean age was 56.7 years. Median Agatston score (IQR) was 0.50 (61.5). Table 1 shows the baseline characteristics by categories of coffee consumption. Subjects who consume more coffee per day were more likely to be active smokers and alcohol drinkers, and less likely to be diabetic.

In crude analysis, higher coffee consumption was associated with higher CAC prevalence [prevalence ratio (95\% CI) of: 1.0 (reference), 1.34 (0.92-1.94), 2.48 (1.81-3.40), 2.45 (1.82-3.30), and 1.81 (1.29-2.56) for coffee consumption of almost never, <1/day, 1/day, 2 $3 /$ day, and $\geq 4$ cups/day respectively ( $\mathrm{p}$ for linear trend $<0.0001$, Table 2 )]. However, adjustment for age, sex, BMI, smoking, alcohol, physical activity, field center, and energy intake eliminated the observed association [corresponding prevalence ratios were 1.0 (reference), 0.92 (0.57-1.49), 1.34 (0.86-2.08), 1.30 (0.84-2.02), and 0.99 (0.60-1.64) respectively ( $\mathrm{p}$ for linear trend, 0.44 , Table 2)], with age and smoking accounting the most attenuation (Table 2). In a sensitivity analysis, there was no evidence of association between coffee consumption and prevalent CAC in either men or women, and when CAC cut points of $0,50,150,200$, and 300 were used.

\section{Discussion}

In our study, we did not find an association between coffee consumption and prevalent CAC in adult subjects free of prevalent CHD after adjustment for cardiovascular risk factors.

The results from our study are comparable to the CARDIA study $(\mathrm{n}=5115)$, where habitual coffee consumption during young adulthood (mean age: 25.6 years) was not associated with prevalent CAC measured 15-20 years later or its progression over 5 years of follow-up [7]. Subjects in the CARDIA study were younger who consumed less coffee (16\% reported consuming 3 cups/day or more). In contrast to the CARDIA study, subjects in our study are middle aged men and women, and hence are representative of general population. 
Contrary to our findings, two studies have shown that consumption of 3-4 cups of coffee per day is associated with lower risk of CAC. In Rotterdam study $(n=1570)$, the Van Woudenbergh et al. showed that coffee consumption of 3-4 cups per day was associated with 59\% lower risk for CAC in women (OR 0.41; 95\% CI 0.25-0.65), but not in men [5]. In sex-specific sensitivity analysis in our study, coffee consumption was not associated with prevalent CAC in either men or women. In Choi et al. study $(n=25138)$, coffee consumption of 3-4 cups per day was associated with $41 \%$ lower prevalence of CAC (score ratio 0.59 ; $95 \%$ CI $0.38-0.93$ ) [6]. In this study, the average age of study participants was 41 years, $83.7 \%$ participants were male, and about $82 \%$ participants have completed college graduation or higher [6]. Hence, their results might not be generalizable to the other populations. Also, compared to using the CAC cut point of 0 and 400 respectively in Choi et al. and Van Woudenbergh et al. studies, our study was robust to use different cut points of 0 , $50,100,150,200$, and 300 to define the presence of coronary calcification.

The mechanism behind potential beneficial relationship between coffee consumption and cardiovascular risk remains unclear. It might be possible that the protective effect of coffee consumption comes through a different physiology than that through reducing atherosclerosis, such as by reducing risk factors responsible for cardiovascular disease. Coffee consumption has been associated with a lower risk of diabetes type 2 [15], improvement of insulin sensitivity [16], hypertension [17], and decreased LDL-C oxidation [18], primarily due to its anti-inflammatory [19] and anti-oxidant [20] properties.

Our study has limitations including its observational cross-sectional design, self-reported dietary and coffee consumption only at baseline which could have limited our ability to capture variations in dietary habits over time affecting the CAC outcomes. Although CAC measurement was completed about 7 years after dietary assessment, we did not have baseline CAC measurement to differentiate calcification that was present at baseline from calcification that developed after assessment of coffee consumption. On the other hand, the large sample size, detailed dietary questionnaire, availability of data on major CHD risk factors, and usage of different cut points of CAC to define presence of coronary calcification are major strengths of the study.

\section{Conclusion}

In conclusion, we found no association between coffee consumption and prevalent CAC in adult men and women.

\section{Acknowledgments}

Sources of funding

This study was supported by grants from the National Heart, Lung, \& Blood Institute (U01 HL067901, U01 HL067897, U01 HL067896, U01 HL067894, U01 HL067893, U01 HL067900, and U01 HL067899).

All authors provided critical revisions for content and had responsibility for the final content. We are indebted to the participants who volunteered in the NHLBI FHS for their outstanding commitment and cooperation and to the entire FHS staff for their expert and unfailing assistance. 


\section{References}

1. [Accessed 17 February 2016] Americans' coffee consumption. at http://www.gallup.com/poll/ 184388/americans-coffee-consumption-steady-few-cut-back.aspx

2. Crippa A, Discacciati A, Larsson SC, Wolk A, Orsini N. Coffee consumption and mortality from all causes, cardiovascular disease, and cancer: a dose-response meta-analysis. Am J Epidemiol. 2014; 180:763-75. [PubMed: 25156996]

3. Greenland P, Bonow RO, Brundage BH, Budoff MJ, Eisenberg MJ, Grundy SM, et al. ACCF/AHA 2007 clinical expert consensus document on coronary artery calcium scoring by computed tomography in global cardiovascular risk assessment and in evaluation of patients with chest pain: a report of the American College of Cardiology Foundation Clinical Expert Consensus Task Force (ACCF/AHA Writing Committee to Update the 2000 Expert Consensus Document on Electron Beam Computed Tomography). Circulation. 2007; 115:402-26. [PubMed: 17220398]

4. Haberl R, Becker A, Leber A, Knez A, Becker C, Lang C, et al. Correlation of coronary calcification and angiographically documented stenoses in patients with suspected coronary artery disease: results of 1,764 patients. J Am Coll Cardiol. 2001; 37:451-7. [PubMed: 11216962]

5. van Woudenbergh GJ, Vliegenthart R, van Rooij FJ, Hofman A, Oudkerk M, Witteman JC, et al. Coffee consumption and coronary calcification: the Rotterdam Coronary Calcification study. Arterioscler Thromb Vasc Biol. 2008; 28:1018-23. [PubMed: 18323515]

6. Choi Y, Chang Y, Ryu S, Cho J, Rampal S, Zhang Y, et al. Coffee consumption and coronary artery calcium in young and middle-aged asymptomatic adults. Heart. 2015; 101:686-91. [PubMed: 25732752]

7. Reis JP, Loria CM, Steffen LM, Zhou X, van Horn L, Siscovick DS, et al. Coffee, decaffeinated coffee, caffeine, and tea consumption in young adulthood and atherosclerosis later in life: the CARDIA study. Arterioscler Thromb Vasc Biol. 2010; 30:2059-66. [PubMed: 20616310]

8. Higgins M, Province M, Heiss G, Eckfeldt J, Ellison RC, Folsom AR, et al. NHLBI family heart study: objectives and design. Am J Epidemiol. 1996; 143:1219-28. [PubMed: 8651220]

9. Djousse L, Arnett DK, Carr JJ, Eckfeldt JH, Hopkins PN, Province MA, et al. Dietary linolenic acid is inversely associated with calcified atherosclerotic plaque in the coronary arteries: the National Heart, Lung, and Blood Institute Family Heart study. Circulation. 2005; 111:2921-6. [PubMed: 15927976]

10. Willett WC, Sampson L, Stampfer MJ, Rosner B, Bain C, Witschi J, et al. Reproducibility and validity of a semiquantitative food frequency questionnaire. Am J Epidemiol. 1985; 122:51-65. [PubMed: 4014201]

11. Rimm EB, Giovannucci EL, Stampfer MJ, Colditz GA, Litin LB, Willett WC. Reproducibility and validity of an expanded self-administered semi-quantitative food frequency questionnaire among male health professionals. Am J Epidemiol. 1992; 135:1114-26. discussion 27-36. [PubMed: 1632423]

12. Stein AD, Shea S, Basch CE, Contento IR, Zybert P. Consistency of the Willett semiquantitative food frequency questionnaire and 24-hour dietary recalls in estimating nutrient intakes of preschool children. Am J Epidemiol. 1992; 135:667-77. [PubMed: 1580243]

13. Carr JJ, Nelson JC, Wong ND, McNitt-Gray M, Arad Y, Jacobs DR Jr, et al. Calcified coronary artery plaque measurement with cardiac CT in population-based studies: standardized protocol of Multi-Ethnic Study of Atherosclerosis (MESA) and Coronary Artery Risk Development in Young Adults (CARDIA) study. Radiology. 2005; 234:35-43. [PubMed: 15618373]

14. Hoffmann U, Brady TJ, Muller J. Cardiology patient page. Use of new imaging techniques to screen for coronary artery disease. Circulation. 2003; 108:e50-3. [PubMed: 12939244]

15. Yarmolinsky J, Mueller NT, Duncan BB, del Bisi Molina MC, Goulart AC, Schmidt MI. Coffee consumption, newly diagnosed diabetes, and other alterations in glucose homeostasis: a crosssectional analysis of the longitudinal study of adult health (ELSA-Brasil). PloS One. 2015; 10:e0126469. [PubMed: 25978631]

16. Loopstra-Masters RC, Liese AD, Haffner SM, Wagenknecht LE, Hanley AJ. Associations between the intake of caffeinated and decaffeinated coffee and measures of insulin sensitivity and beta cell function. Diabetologia. 2011; 54:320-8. [PubMed: 21046357] 
17. Grosso G, Stepaniak U, Polak M, Micek A, Topor-Madry R, Stefler D, et al. Coffee consumption and risk of hypertension in the Polish arm of the HAPIEE cohort study. Eur J Clin Nutr. 2015; 70:109-15. [PubMed: 26220566]

18. Yukawa GS, Mune M, Otani H, Tone Y, Liang XM, Iwahashi H, et al. Effects of coffee consumption on oxidative susceptibility of low-density lipoproteins and serum lipid levels in humans. Biochem Biokhimiia. 2004; 69:70-4.

19. Cardenas C, Quesada AR, Medina MA. Anti-angiogenic and anti-inflammatory properties of kahweol, a coffee diterpene. PloS One. 2011; 6:e23407. [PubMed: 21858104]

20. Richelle M, Tavazzi I, Offord E. Comparison of the antioxidant activity of commonly consumed polyphenolic beverages (coffee, cocoa, and tea) prepared per cup serving. J Agric Food Chem. 2001; 49:3438-42. [PubMed: 11453788] 


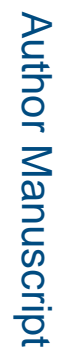

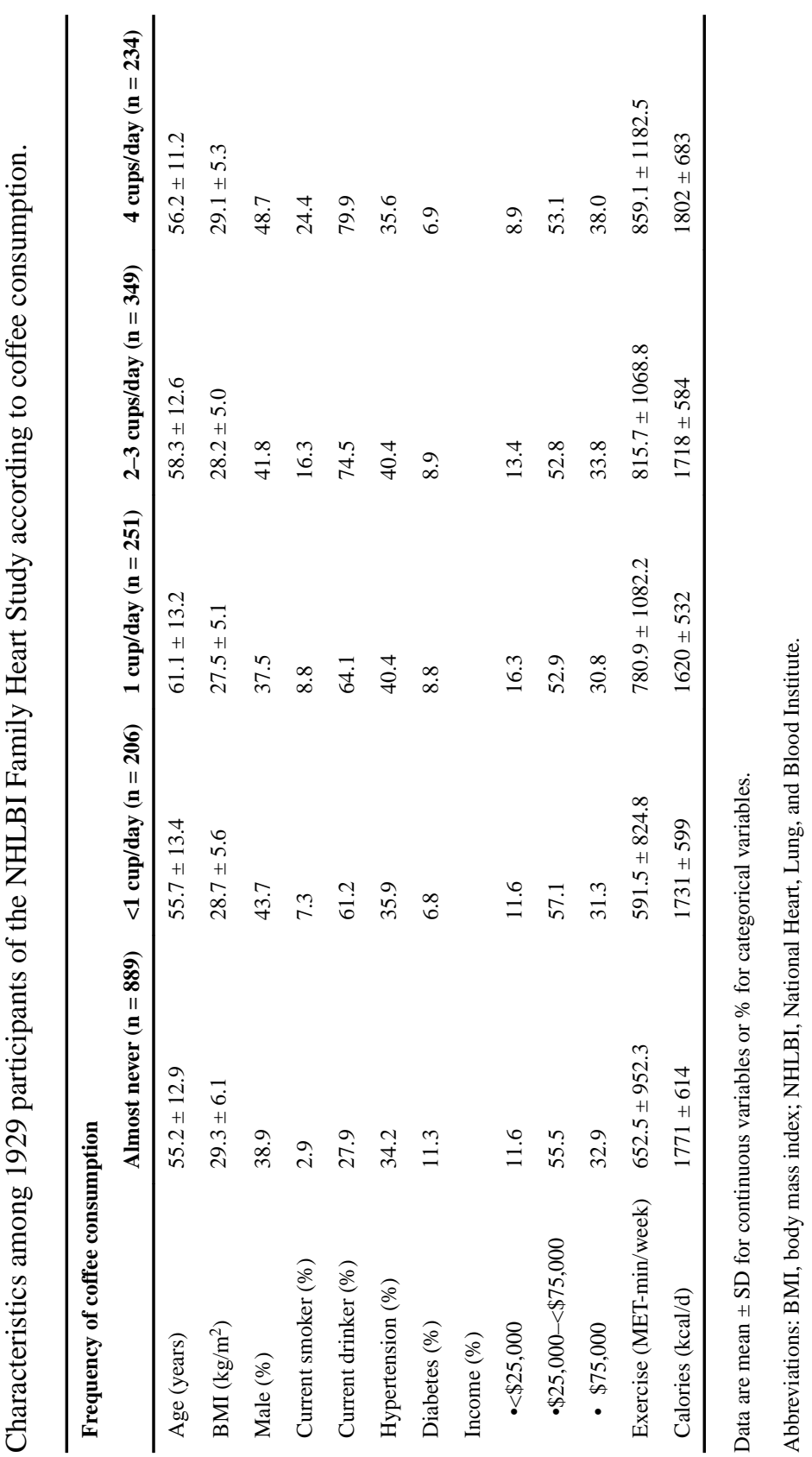

Clin Nutr ESPEN. Author manuscript; available in PMC 2017 July 07. 


\section{Table 2}

Prevalence ratio (95\% confidence intervals) of calcified atherolsclerotic plaque in the coronary arteries according to coffee consumption in 1929 participants in the NHLBI Family Heart Study.

\begin{tabular}{lllll}
\hline Frequency of coffee consumption & CAC prevalence (\%) & Crude & Model 1 $^{\boldsymbol{a}}$ & Model $^{\boldsymbol{b}}$ \\
\hline Almost never & 16.8 & 1.00 & 1.00 & 1.00 \\
$<1$ cup/day & 21.4 & $1.34(0.92-1.94)$ & $1.03(0.67-1.58)$ & $0.92(0.57-1.49)$ \\
1 cup/day & 33.5 & $2.48(1.81-3.40)$ & $1.32(0.89-1.94)$ & $1.34(0.86-2.08)$ \\
$2-3$ cups/day & 33.2 & $2.45(1.82-3.30)$ & $1.53(1.07-2.18)$ & $1.30(0.84-2.02)$ \\
$\geq 4$ cups/day & 26.9 & $1.81(1.29-2.56)$ & $1.21(0.77-1.90)$ & $0.99(0.60-1.64)$ \\
$P$ for trend & & $<0.0001$ & 0.17 & 0.44 \\
\hline
\end{tabular}

Abbreviations: CAC, coronary artery calcium; NHLBI, National Heart, Lung, and Blood Institute.

${ }^{a}$ Model 1 is adjusted for age and smoking.

${ }^{b}$ Model 2 is adjusted for age, sex, BMI, smoking, alcohol, physical activity, field center, and total calories. 